\title{
Distúrbios gastrointestinais no transtorno do espectro autista: revisão integrativa
}

\author{
Gastrointestinal disorders in autism spectrum disorder: an integrative review \\ Trastornos gastrointestinales en el trastorno del especto autista: una revisión integradora
}

Recebido: 16/11/2021 | Revisado: 21/11/2021 | Aceito: 22/11/2021 | Publicado: 03/12/2021

\author{
Bianca Ferreira de Sousa \\ ORCID: https://orcid.org/0000-0001-9700-0100 \\ Centro Universitário Santo Agostinho, Brasil \\ E-mail: biiancafs@gmail.com \\ Jéssica Carvalho Santos Moura \\ ORCID: https://orcid.org/0000-0002-4452-2463 \\ Centro Universitário Santo Agostinho, Brasil \\ E-mail: jessikarvalho09@gmail.com \\ Luiza Marly Freitas de Carvalho \\ ORCID: https://orcid.org/0000-0002-6726-3994 \\ Centro Universitário Santo Agostinho, Brasil \\ E-mail: lumarnahid@gmail.com \\ Kamylla Monteiro Moraes \\ ORCID: https://orcid.org/0000-0002-1863-5384 \\ Hospital Getúlio Vargas, Brasil \\ E-mail: kamyllaamore1@gmail.com
}

\begin{abstract}
Resumo
O Transtorno do Espectro Autista (TEA) é uma síndrome que afeta crianças na primeira infância e não tem cura. Nos indivíduos com TEA, os distúrbios gastrointestinais podem agravar questões comportamentais especialmente pela dificuldade de comunicação, podendo prejudicar a aprendizagem e a socialização. O presente trabalho se justifica pelo aumento da incidência de distúrbios alimentares em pessoas com TEA, sendo necessário entender como certas escolhas dietéticas estão contribuindo para o desenvolvimento destas e quais outros fatores podem estar relacionados. Dessa forma, pretende-se entender a relação entre os distúrbios alimentares e o autismo. Realizou-se uma revisão integrativa sobre distúrbios alimentares em crianças portadoras de TEA, foram selecionados 15 artigos para análise utilizando os anos de 2010 a 2021. Observou-se que o glúten e a caseína podem ter um importante impacto na vida dos portadores de TEA, uma vez que podem estar associados à danos às microvilosidades intestinais, contribuindo para má absorção destas substâncias e desconforto gastrointestinal. Conclui-se que é de grande importância para crianças com TEA a introdução de uma intervenção dietética para a melhora da saúde física e mental, uma dieta equilibrada tem um impacto favorável na saúde mental e o bem-estar desses indivíduos.
\end{abstract}

Palavras-chave: Gastroenteropatias; Autismo; Criança; Nutrição da criança; Transtorno do espectro autista (TEA).

\begin{abstract}
Autistic Spectrum Disorder (ASD) is a syndrome that affects children in infancy and cannot be cured. In individuals with ASD, gastrointestinal disorders can aggravate behavioral issues, especially due to communication difficulties, which can impair learning and socialization. The present work is justified by the increased incidence of eating disorders in people with ASD, it is necessary to understand how certain dietary choices are contributing to their development and what other factors may be related. Thus, it is intended to understand the relationship between eating disorders and autism. An integrative review of eating disorders in children with ASD was carried out, 15 articles were selected for analysis using the years 2010 to 2021. It was observed that gluten and casein can have an important impact on the lives of patients with ASD, since they may be associated with damage to intestinal microvilli, contributing to poor absorption of these substances and gastrointestinal discomfort. It is concluded that it is of great importance for children with ASD to introduce a dietary intervention to improve physical and mental health, a balanced diet has a favorable impact on the mental health and well-being of these individuals.
\end{abstract}

Keywords: Gastrointestinal diseases; Autism; Child; Child nutrition; Autistic spectrum disorder (ASD).

\section{Resumen}

El trastorno del espectro autista (TEA) es un síndrome que afecta a los niños en la infancia y no se puede curar. En las personas con TEA, los trastornos gastrointestinales pueden agravar los problemas de comportamiento, especialmente debido a las dificultades de comunicación, que pueden afectar el aprendizaje y la socialización. El presente trabajo se justifica por la mayor incidencia de trastornos alimentarios en personas con TEA, es necesario comprender cómo determinadas elecciones dietéticas están contribuyendo a su desarrollo y qué otros factores pueden estar relacionados. Por lo tanto, se pretende comprender la relación entre los trastornos alimentarios y el autismo. Se realizó una revisión 
integradora de los trastornos alimentarios en niños con TEA, se seleccionaron 15 artículos para su análisis utilizando los años 2010 a 2021. Se observó que el gluten y la caseína pueden tener un impacto importante en la vida de los pacientes con TEA, ya que pueden estar asociado con daño a las microvellosidades intestinales, contribuyendo a una mala absorción de estas sustancias y malestar gastrointestinal. Se concluye que es de gran importancia para los niños con TEA introducir una intervención dietética para mejorar la salud física y mental, una dieta balanceada tiene un impacto favorable en la salud mental y el bienestar de estos individuos.

Palabras clave: Enfermedades gastrointestinales; Autismo; Niño; Nutrición del niño; Trastorno del espectro autista (TEA).

\section{Introdução}

Várias concepções sobre as crianças foram se constituindo ao longo do tempo, as quais contribuíram para que se construísse um olhar de cuidado sobre as mesmas e com a infância. Crianças são curiosas, tudo que vêem querem tatear, ver de perto, sentir o cheiro e até colocarem na boca para descobrirem se esse "algo novo" tem sabor. As crianças com autismo, de forma geral, possuem déficits na comunicação, interação social, estereótipos de comportamento e sensoriais, que se apresentam antes dos três anos de idade, isso requer que as atividades de vida diária sejam ajustadas às suas necessidades e demandas (Minatel \& Matsukura, 2014).

Conforme a Organização Mundial da Saúde (OMS), em 2017, a cada 160 crianças no mundo, uma nasce com autismo e, para o Brasil, indica-se que 1\% da população, cerca de 2 milhões de pessoas, estejam dentro do TEA, tendo maior incidência no sexo masculino.

Cada ser humano tem características pessoais, psicológicas e corporais específicas, mas no TEA há evidências sobre os benefícios da nutrição para melhoria da qualidade de vida do indivíduo, que além da seletividade alimentar, comumente apresenta problemas gastrointestinais associados. Embora estudos científicos sobre o autista e sua alimentação venham auxiliando o progresso da qualidade de vida, com reflexo na melhoria dos comportamentos e atitudes próprias destas crianças, ainda não há consenso entre os pesquisadores. (Souza et al., 2019).

Estudos apontam que as modificações gastrointestinais podem ter atuação na patogênese e prognóstico do TEA. Evidências crescentes propõem que a microbiota é mudada em resposta a fatores de risco etiológicos para TEA (Pinho \& Silva, 2011). Além da alta prevalência de distúrbios do TGI em crianças com TEA, já foi evidenciado que as chances destes distúrbios acontecerem são quatro vezes maiores do que em crianças neurotípicas. Os sintomas mais frequentes são constipação, diarreia, síndrome do intestino irritável, aumento da permeabilidade intestinal, falta de apetite e disbiose intestinal (McElhanon et al., 2014; Iovene et al., 2017).

Os sintomas gastrointestinais tornam-se uma situação desafiadora no cuidado do paciente autista, visto que sua interpretação é prejudicada pela dificuldade comunicativa referente ao transtorno, podendo ser precipitados ou agravados por episódios de fuga à rotina do mesmo, como casos de constipação ocorridos por causa das mudanças ou frustrações em seus hábitos fisiológicos (McElhanon et al., 2014).

Certos estudos indicam que intervenções nutricionais podem ser uma via de tratamento dos sintomas comportamentais e gastrointestinais no TEA. Entretanto, não existe ainda embasamento científico para explicar certas restrições alimentares, podendo até causar danos nutricionais, em razão da maior seletividade alimentar demonstrada por crianças com TEA (Peretti, et al., 2018).

O presente estudo trata-se de uma revisão integrativa que tem por objetivo entender a relação entre os distúrbios gastrointestinais e o autismo. 


\section{Metodologia}

Para o desenvolvimento e conclusão de algo é necessária a realização de pesquisa, com o objetivo de obter descobertas acerca da temática escolhida. Para Marconi e Lakatos (2011) a finalidade da pesquisa é descobrir respostas para questões, aplicando métodos científicos. A realização do presente estudo se deu através de revisão integrativa de literatura, que permitiu uma abordagem ampla, a partir dos estudos analisados. A revisão integrativa é cientificamente reconhecida, pois permite, a partir da síntese de estudos publicados, a inclusão de literatura teórica e empírica, bem como outros estudos com abordagens quantitativas e/ou qualitativas, permitindo discussões relacionadas ao tema em estudo. Além disso, fornece informações para a definição de conceitos, revisão de teorias e/ou análise metodológica, resultando em contribuições para a ciência e para a prática clínica (Ercole; Melo \& Alcoforado, 2014).

Por se tratar de uma pesquisa de cunho integrativo, foi realizada uma busca, nos meses de setembro a novembro, em publicações/artigos, considerando produções dos últimos dez anos (2011-2021), utilizando como base de dados as seguintes plataformas: Biblioteca Virtual de Saúde (BVS), Scientific Electronic Library Online (SciELO), National Library of Medicine (Pubmed) e Google Acadêmico. Serão manuseados publicações/artigos nas línguas: Português, Inglês e Espanhol, tendo como descritores: gastroenteropatias and autismo and criança and nutrição da criança and Transtorno do Espectro Autista (TEA). Os critérios para a escolha das palavras-chave consistiram em: pertencer aos Descritores em Ciências da Saúde (DeCS) e representar ao menos em parte a temática do estudo.

A partir do processo acima mencionado será possível investigar a relação entre TEA e distúrbios gastrointestinais, relacionando, descrevendo e associando fatores desencadeadores e os alimentos envolvidos, caracterizando os sujeitos da pesquisa.

Os critérios de inclusão foram: estudos experimentais e randomizados, publicados em inglês, português e espanhol, desenvolvidos com seres humanos em todas as faixas etárias no TEA, no período compreendido de 2010 e 2021 . Os critériosde exclusão foram publicações que não atendam aos descritores (gastroenteropatias and autismo and criança and nutrição da criança and transtorno do espectro autista) de forma total ou parcial e estudos que não foram disponibilizados na integra.

A pesquisa for realizada por meio da análise de 10 artigos, a partir do resumo e do título, sendo incluídos, assim, aqueles que possuem relação com os descritores de pesquisa. Em seguida, foi realizada uma leitura minuciosa e crítica dos manuscritos para identificação dos núcleos de sentido de cada texto e posterior agrupamento de subtemas que sintetizem as produções.

\section{Resultados e Discussão}

Mediante os critérios de inclusão e exclusão de artigos, foram selecionados 456 artigos para a presente revisão. Após a inclusão dos descritores: espectro autista, alimentação infantil, alergia alimentar e hipersensibilidade alimentar no espectro autista, foram selecionados 108 artigos, utilizando os anos de 2011 a 2021. Após uma leitura minuciosa do resumo, foram selecionados 10 artigos para análise.

No Quadro 1 estão os resultados que abordam a caracterização do TEA. 
Quadro 1: Caracterização do TEA.

\begin{tabular}{|c|c|c|}
\hline AUTOR/ANO & TÍTULO & RESULTADOS \\
\hline $\begin{array}{l}\text { American } \\
\text { Psychiatric } \\
\text { Association } \\
(2013)\end{array}$ & $\begin{array}{l}\text { Manual diagnóstico e } \\
\text { estatístico de Transtornos } \\
\text { mentais }\end{array}$ & $\begin{array}{l}\text { O TEA é uma desordem no neurodesenvolvimento que engloba o transtorno autista (autismo), } \\
\text { a Síndrome de Rett, o Transtorno de Asperger, o transtorno desintegrativo da infância e o } \\
\text { transtorno global do desenvolvimento sem outra especificação. Na atualidade, } 1 \% \text { da } \\
\text { população mundial é diagnosticada com TEA. A prevalência desse transtorno, nos Estados } \\
\text { Unidos, é de uma a cada } 68 \text { crianças com oito anos de idade. }\end{array}$ \\
\hline $\begin{array}{l}\text { Jacquemont et } \\
\text { al. } \\
(2014)\end{array}$ & $\begin{array}{l}\text { A higher mutational } \\
\text { burden in females } \\
\text { supports a "female } \\
\text { protective model" in } \\
\text { neurodevelopmental } \\
\text { disorders. }\end{array}$ & $\begin{array}{l}\text { Demonstrou predomínio do autismo em indivíduos do gênero masculino, examinando } \\
\text { diferenças de bases moleculares entre meninos (n=9206) e meninas (n=6379) no } \\
\text { neurodesenvolvimento. Contudo, mesmo que os dados mostrem certo "modelo protetor" no } \\
\text { sexo feminino, é fundamental que se faça mais replicações deste estudo para certificar o que } \\
\text { foi concluído. }\end{array}$ \\
\hline
\end{tabular}

Fonte: Dados da pesquisa (2021).

Os autores revelaram que as meninas com TEA e com alguma alteração no desenvolvimento neurológico, tiveram maior número de mutações de ordem genética. A sua etiologia ainda é desconhecida, apesar de haver várias hipóteses e já ter mais de 70 anos desde os primeiros estudos publicados. Estudos indicam que fatores genéticos e ambientais associam a ocorrência do TEA e cujos sintomas podem interferir na vida social da criança, levá-la de um retardo mental e até uma produtividade intelectual acima da média (Dias et al., 2018).

No Quadro 2 estão distribuídos os resultados abordando a incidência dos distúrbios gastrointestinais no transtorno do espectro autista.

Quadro 2: Distribuição dos resultados sobre incidência de distúrbios gastrointestinais no transtorno do espectro autista (TEA).

\begin{tabular}{|c|c|c|}
\hline AUTOR/ANO & TÍTULO & RESULTADOS \\
\hline $\begin{array}{l}\text { Zhu et al. } \\
\text { (2017) }\end{array}$ & $\begin{array}{l}\text { Association between } \\
\text { behavioral problems } \\
\text { and gastrointestinal } \\
\text { disorders among } \\
\text { children with autism } \\
\text { spectrum disorder }\end{array}$ & $\begin{array}{l}\text { Crianças com TEA retratam maior risco de distúrbios gastrointestinais. Comportamentos } \\
\text { estereotipados, conturbados e emocionais são mais graves nas crianças com TEA com } \\
\text { problemas gastrointestinais. }\end{array}$ \\
\hline $\begin{array}{l}\text { Caetano \& Gurgel } \\
\text { (2018) }\end{array}$ & $\begin{array}{l}\text { Perfil nutricional de } \\
\text { crianças portadoras do } \\
\text { espectro autista }\end{array}$ & $\begin{array}{l}\text { Além das características que as crianças com TEA apresentam, percebeu-se ainda muitas } \\
\text { desordens gastrointestinais, como redução da produção de enzimas digestivas, inflamação } \\
\text { da parede intestinal e permeabilidade intestinal alterada. Esses sintomas incluem diarreia } \\
\text { persistente, obstipação, gases, inchaço abdominal, desconforto abdominal e regurgitação, } \\
\text { o que pode ampliar a irritabilidade e, consequentemente, problemas comportamentais. }\end{array}$ \\
\hline $\begin{array}{l}\text { Silva, Santos \& Silva } \\
(2020)\end{array}$ & $\begin{array}{l}\text { Excesso de peso e } \\
\text { sintomas } \\
\text { gastrintestinais em um } \\
\text { grupo de crianças } \\
\text { autistas }\end{array}$ & $\begin{array}{l}\text { Diante do cenário de elevada prevalência de modificações gastrintestinais, foram } \\
\text { relacionadas evidências que vinculam a relação da disbiose intestinal com as } \\
\text { modificações gastrintestinais e neurológicas nas crianças com TEA. A conservação do } \\
\text { aleitamento materno traz benefício adicional no final do primeiro e segundo anos de vida, } \\
\text { por sua presença no lúmen intestinal estimular o desenvolvimento de sua mucosa e da } \\
\text { atuação da enzima lactase, protegendo assim de alterações gastrintestinais. }\end{array}$ \\
\hline
\end{tabular}




\begin{tabular}{|c|c|c|}
\hline $\begin{array}{l}\text { Harry \& Cardy } \\
(2012)\end{array}$ & $\begin{array}{l}\text { A pilot study evaluate } \\
\text { nutricional influences } \\
\text { on gastrointestinal } \\
\text { symptoms and } \\
\text { behavior patterns in } \\
\text { children with Autism } \\
\text { Spectrum Disorder. }\end{array}$ & $\begin{array}{l}\text { Os distúrbios gastrointestinais parecem estar relacionados à intensificação de problemas } \\
\text { comportamentais em crianças com TEA, devido a ocorrência de refluxo exofágico, dores } \\
\text { abdominais, diarréias, constipação, edema, etc., os quais podem estar associados a } \\
\text { comprometimentos da microbiota intestinal, que com crescimento de microrganismos } \\
\text { patogênicos que podem produzir neurotoxinas. Esses sintomas podem ser relacionados a } \\
\text { uma ruptura na mucosa do intestino, ocorrendo absorção inadequada de proteínas, como a } \\
\text { gliadina e caseína, as quais possuem potencial para desencadear processos inflamatórios, } \\
\text { pois são percursores de neuropeptídios que modificam a função neurológica. }\end{array}$ \\
\hline $\begin{array}{l}\text { Fulceri et al. } \\
\text { (2016) }\end{array}$ & $\begin{array}{l}\text { Gastrointestinal } \\
\text { symptoms and } \\
\text { behavioral problems in } \\
\text { preschoolers with } \\
\text { autism spectrum } \\
\text { disorder }\end{array}$ & $\begin{array}{l}\text { Em } 281 \text { crianças observou-se, em avaliação comparativa, que aquelas com TEA têm } \\
\text { chances } 22,6 \% \text { maiores de sofrerem com disfunções gastrointestinais, principalmente } \\
\text { constipação e falta de apetite, e de intensidade mais grave do que crianças de } \\
\text { neurodesenvolvimento normal. Além disso, observaram que crianças com TEA com } \\
\text { disfunções gastrointestinais apresentam problemas mais acentuados de irritabilidade, } \\
\text { ansiedade, queixas somáticas, isolamento social, comportamentos externalizantes } \\
\text { (comportamentos desafiadores de oposição e birras), sensibilidade sensorial, problemas } \\
\text { de sono e comportamentos rígido-compulsivos. }\end{array}$ \\
\hline
\end{tabular}

Fonte: Dados da pesquisa (2021).

A identificação de alterações do TGI dessas crianças deve ser aumentada, pois poderá reconhecer fatores de risco, reduzindo, assim, o impacto negativo dos sintomas no processo das modificações do desenvolvimento comportamental. Outra questão significativa é a pesquisa de possíveis fatores genéticos relevantes e/ou marcadores biológicos que, se identificados, poderão melhorar a capacidade na categorização clínica e dos subtipos dentro do amplo espectro do autismo. Há, portanto, muitas perguntas, ainda, a serem respondidas nas várias linhas de investigação neste campo (Pinho \& Silva, 2011).

Segundo Mezzelani et al (2015) os sintomas gastrointestinais mais relatados em indivíduos TEA são constipação crônica que pode evoluir com encoprese, dor abdominal com ou sem diarreia, inchaço abdominal, deficiências em dissacaridases, inflamação do trato gastrointestinal e anormalidades do sistema nervoso entérico.

No cenário de prevenir ou melhorar a disbiose, Fernandes (2017) pontuou fatores protetores que já são citados como facilitadores para o desenvolvimento de uma microbiota saudável, como a prática de amamentação exclusiva, parto normal e nascimento a termo.

Indivíduos com TEA são muito seletivos quanto a sua forma de se alimentar que limita a variedade de alimentos, podendo desencadear em carências nutricionais. Desse modo é necessária cautela ao deixar as crianças autistas selecionar e ingerir alimentos que não sejam saudáveis. (Meneses et al, 2018; Rocha et al., 2019).

Na procura de uma compreensão melhor das modificações referentes ao trato gastrointestinal, estudos têm examinado os hábitos alimentares de portadores de TEA, bem como a aplicação de dietas especiais (Lange; Hauser \& Reissmann, 2015).

No Quadro 3 estão dispostos os artigos que abordam questões referentes a alimentação, nutrição e TEA. 
Quadro 3: Distribuição dos resultados referentes a Alimentação, Nutrição e TEA.

\begin{tabular}{|c|c|c|}
\hline AUTOR/ANO & TÍTULO & RESULTADOS \\
\hline $\begin{array}{l}\text { Santos } \\
(2015)\end{array}$ & A Nutrição da Criança Autista & $\begin{array}{l}\text { Com a eliminação padronizada e controlada dos alimentos que promovem a formação } \\
\text { das substâncias similares aos opióides da dieta dos auistas, percebe-se melhora } \\
\text { significativa na sociabilidade e comunicação destes pacientes, bem como, uma redução } \\
\text { dos efeitos de abstinência destes compostos. }\end{array}$ \\
\hline $\begin{array}{l}\text { Mello } \\
(2016)\end{array}$ & Autismo: Guia Prático & $\begin{array}{l}\text { Após o período de experiência em relação à restrição da caseína e derivados, foi } \\
\text { iniciada a retirada do glúten e derivados da alimentação, com o mesmo critério de } \\
\text { observação utilizado com a retirada da caseína, porém por um período maior, de cinco } \\
\text { meses. Ao final, percebeu-se uma melhora nas características e sintomas de desordem } \\
\text { comportamentais e desconfortos gástricos. }\end{array}$ \\
\hline $\begin{array}{l}\text { Carvalho et al. } \\
\text { (2012) }\end{array}$ & $\begin{array}{l}\text { Nutrição e autismo: } \\
\text { Considerações } \quad \text { sobre a } \\
\text { Alimentação do Autista }\end{array}$ & $\begin{array}{l}\text { A retirada do glúten e da caseína da alimentação de crianças autistas promove uma } \\
\text { melhora na qualidade de vida das mesmas, uma vez que diminui os sintomas } \\
\text { gastrointestinais, diminuindo os impulsos hiperativos. }\end{array}$ \\
\hline $\begin{array}{l}\text { Dias et al. } \\
\text { (2018) }\end{array}$ & $\begin{array}{l}\text { Dieta isenta de glúten e } \\
\text { caseína no transtorno do } \\
\text { espectro do autismo: uma } \\
\text { revisão sistemática }\end{array}$ & $\begin{array}{l}\text { Os estudos apontam que o aumento de sintomas comportamentais de pessoas no TEA } \\
\text { pode ser causado pela má digestão de alimentos contendo glúten e caseína, uma vez em } \\
\text { excesso no trato gastrointestinal vão para a corrente sanguínea devido a uma alteração } \\
\text { na permeabilidade da membrana intestinal e por meio da circulação pode afetar o } \\
\text { sistema nervoso central (SNC), se unem a neuropeptídios opióides gerando uma } \\
\text { atividade acentuada e abalando sistemas neurais, o que sucederia na sintomatologia. } \\
\text { Dessa forma, uma maior permeabilidade intestinal em crianças com TEA foi expressa } \\
\text { quando comparada com crianças sem o diagnóstico de TEA. Portanto, uma dieta isenta } \\
\text { de glúten e caseína pode ser um via para melhoria da qualidade de vida das crianças no } \\
\text { TEA. Porém, é necessário mais estudos sobre o assunto. }\end{array}$ \\
\hline $\begin{array}{l}\text { Lange, Hauser e } \\
\text { Reissmann } \\
\text { (2015) }\end{array}$ & $\begin{array}{l}\text { Gluten-free and casein-free } \\
\text { diets in the therapy of autism }\end{array}$ & $\begin{array}{l}\text { Algumas dietas restritivas têm sido correlacionadas com a suavização de sintomas } \\
\text { comportamentais, fora a melhoria dos sintomas gastrointestinais. Neste âmbito, as } \\
\text { dietas que retiram glúten e caseína são utilizadas com mais freqüência. }\end{array}$ \\
\hline
\end{tabular}

Fonte: Dados da pesquisa (2021).

Em diferentes estudos, a porcentagem de crianças com TEA que sofrem de distúrbios gastrointestinais varia, mas esses estudos estão de acordo que anormalidades gastrointestinais são comuns no TEA, e noções sobre o potencial de uma dieta sem glúten, dieta sem caseína ou dieta combinada sem glúten e sem caseína para acometer os sintomas de condições do espectro do autismo persistem por muitos anos. A alergia alimentar e a inflamação do intestino por outras razões podem esclarecer problemas e sintomas nas crianças com autismo por diversos mecanismos (Meneses et al. 2018; Rocha et al., 2019).

Mannion e Leader (2014) demonstram que vários estudos mostram a relação do glúten e caseína como vilã para os autistas muitas vezes apontam alguns incômodos gastrointestinais, que muitas vezes pioram os sintomas comportamentais dos autistas, uma vez que a evidência propõe que existem relações diretas e indiretas entre microbiota, o intestino e o cérebro e ocorrem várias teorias que relatam esta interação no autismo. Uma das teorias é a "disbiose intestinal" que menciona uma alteração da microbiota intestinal.

A dieta sem glúten e sem caseína é justificada em virtude dos efeitos dos peptídeos opióides, que são liberados na digestão dessas proteínas, e com isso reduzem a captação de cisteína pelas células, como conseqüência disso, gera níveis diminuídos de glutationa, pois eles são limitantes para síntese de GSH, ademais reduzem o índice de metilação. Desse modo, essa dieta beneficiaria a absorção de cisteína e consequentemente os níveis de glutationa, que quando está em menor 
quantidade no trato gastrointestinal pode promover inflamação, favorecendo o desconforto no TEA (Lázaro; Pondé \& Rodrigues, 2016).

\section{Conclusão}

Os estudos expuseram que o glúten e a caseína podem ter um considerável impacto na vida dos portadores de TEA, visto que provocam danos as microvilosidades do intestino colaborando para a má absorção tanto do glúten e da caseína quanto de nutrientes, podendo causar desconforto gastrointestinal como diarreias, flatulências que poderiam resultarn a irritabilidade dessas crianças.

A nutrição e os fatores ambientais exercem papéis importantes para a melhoria na qualidade de vida dessas crianças. Os inúmeros estudos científicos sobre alimentação do indivíduo autista, que se associam à experiência de pessoas diretamente comprometidas, parecem auxiliar para a melhora do comportamento e desconforto gastrointestinal de crianças com diagnóstico de TEA.

É de grande importância para crianças com TEA a introdução de uma intervenção dietética para a melhora da saúde física e mental. As pesquisas mostram que a dieta correta tem um impacto benigno na saúde mental e o bem-estar, em especial na sintomatologia que gera muitas oportunidades para a melhora da qualidade de vida desses indivíduos. A pluralidade dos estudos publicados exibe alterações positivas na apresentação dos sintomas após a introdução de uma intervenção dietética. Principalmente melhorias na comunicação, atenção e, ainda, diminuição da hiperatividade.

Apesar das evidências científicas atualmente disponíveis, notou-se a dificuldade de estabelecer medidas adequadas para considerar uma resposta positiva nas sintomatologias funcionais, recomendando que mais estudos sejam realizados para investigar quais seriam as melhores abordagens em intervenções dietéticas, por isso é necessário ampliar essas produções para poder servir de contribuição para os profissionais agirem de forma efetiva em pessoas com TEA.

\section{Referências}

American Psychiatric Association. Diagnostic and Statistical Manual of Mental disorders. (2013). DSM-5. Washington (USA): American Psychiatric Association.

Caetano, M., \& Gurgel, D. (2018). Perfil nutricional de crianças portadoras do espectro autista. Rev Bras Promoção da Saúde, Fortaleza, 31(1), 1-11.

Carvalho, J. A., Santos, C. S. S., Carvalho, M. P., \& Souza, L. S. (2012). Nutrição e Autismo: Considerações sobre a Alimentação do Autista. Revista Científica do ITPAC, Araguaína, 5(1), 32-65.

Dias, E. C., Rocha, J. S., Ferreira, G. B., \& Pena, G. G. (2018). Dieta isenta de glúten e caseína no transtorno do espectro do autismo: uma revisão sistemática. Revista Cuidarte, 9(1), 1-16.

Ercole, F. F., Melo, L. S., \& Alcoforado, C. L. G. C. (2014). Revisão Integrativa versus Revisão Sistemática. Revista Brasileira de Enfermagem, Brasília, $18(1), 9-11$

Fernandes, T. F. (2017). Impactos da microbiota intestinal na saúde do lactente e da criança em curto e longo prazo. International Journal of Nutrology, 10(1), $335-342$.

Fulceri, F., Morelli, M., Santocchi, E., Cena, H., Bianco, T. D., Narzisi, A., Calderoni, S., \& Muratori, F. (2016). Gastrointestinal symptoms and behavioral problems in preschoolers with autism spectrum disorder. Digestive and Liver Disease, 48(3), 248-254.

Harry, C., \& Cardy, B. (2012). A pilot study evaluate nutricional influences on gastrointestinal symptoms and behavior patterns in children with Autism Spectrum Disorder. Complementary Therapies in Medicine. 20(6), 437-440.

Iovene, M. R., Bombace, F., Maresca, R., Sapone, A., Iardino, P., Picardi, A., Marotta, R., Schiraldi, C., Siniscalco, D., Serra, N., Magistris, L., \& Bravaccio, C. (2017). Intestinal dysbiosis and yeast isolation in stool of subjects with autism spectrum disorders. Mycopathologia, Califórnia, 182(3-4), $349-363$.

Jacquemont, S., Coe, B. P., Hersch, M., Duyzend, M. H., Krumm, N., Bergmann, S., Beckmann, J. S., Rosenfeld, J. A., \& Eichler, E. E. (2014). A higher mutational burden in females supports a "female protective model" in neurodevelopmental disorders. The American Journal of Human Genetics, 94(30, 415425 .

Lange, K. W., Hauser, J., \& Reissmann, A. (2015). Gluten-free and casein-free diets in the therapy of autism. Current Opinion in Clinical Nutrition \& Metabolic Care, 18(6), 572-575. 
Research, Society and Development, v. 10, n. 15, e536101523375, 2021

(CC BY 4.0) | ISSN 2525-3409 | DOI: http://dx.doi.org/10.33448/rsd-v10i15.23375

Lázaro, C. P., Pondé, M. P., \& Rodrigues, L. E. A. (2016). Opioid peptides and gastrointestinal symptoms in autism spectrum disorders. Revista Brasileira de Psiquiatria, 38(3), 243-246.

Mannion, A., \& Leader, G. (2013). Comorbidity in autism spectrum disorder: A literature review. Research in Autism Spectrum Disorders, 7(12), 1595-1616.

Marconi, M. A., \& Lakatos E. M. (2011). Técnicas de pesquisa: planejamento e execução de pesquisas, amostragens e técnicas de pesquisa, elaboração e interpretação de dados. São Paulo: Atlas, (7a ed.,) p. 277.

McElhanon, B. O., McCraken, C., Karpen, S., \& Sharp, W. D. (2014). Gastrointestinal symptoms in Autism Spectrum disorder: a meta-analysis. Pediatrics, São Paulo, 133(5), 872-883.

Mello, A. M. S. R. (2016). Autismo: Guia Prático, (8 ed.): CORDE.

Meneses, R. D. C. G., Santos, I. S., Teixeira, K. L., de Almeida, M. S., \& Fernandes, M. C. O. (2018). Interferência alimentar na capacidade cognitiva do indivíduo com transtorno espectro autista. VI Encontro Internacional de Jovens Investigadores. Editora Realize.

Mezzelani, A., Landini, M., Facchiano, F., Raggi, M. E., Villa, L., Molteni, M., \& Marabotti, A. (2015). Environment, dysbiosis, immunity and sex-specific susceptibility: a translational hypothesis for regressive autism pathogenesis. Nutritional neuroscience, 18(4), $145-161$.

Minatel, M. M., \& Matsukura, T. S. (2014). Famílias de crianças e adolescentes com autismo: Cotidiano e realidade de cuidados em diferentes etapas do desenvolvimento. Revista de Terapia Ocupacional da Universidade de São Paulo, 25(2), 126-34.

Peretti, S., Mariano, M., Mazzocchetti, C., Mazza, M., Pino, M. C., Verrotti Di Pianella, A., \& Valenti, M. (2019). Diet: the keystone of autism spectrum disorder?. Nutritional neuroscience, 22(12), 825-839.

Pinho, M. A., \& Silva, L. R. (2011). Manifestações digestórias em portadores de transtornos do espectro autístico necessidade de ampliar as perguntas e respostas. Revista De Ciências Médicas E Biológicas, 10(3), 304-309.

Rocha, G. S. S., de Medeiros Júnior, F. C., Lima, N. D. P., da Silva Machado, A., Pereira, I. C., da Silva Lima, M. \& da Silva, H. A. C. (2019). Análise da seletividade alimentar de pessoas com Transtorno do Espectro Autista. Revista Eletrônica Acervo Saúde, v. 24 , p. 538.

Santos, C. A. B. (2015). A Nutrição da Criança Autista. Belo Horizonte: UNIFENAS.

Silva, D. V., Santos, P. N. M., \& Silva, D. A. V. (2020). Excesso de peso e sintomas gastrintestinais em um grupo de crianças autistas. Revista Paulista de Pediatria, v. 1, p. 1-15.

Souza, J. V. S., Patrício, W. T. R., Zambom, T. R. L., \& Batista, S. L. (2019). Autismo e transtorno alimentar: a inclusão e a capacidade de aceitação das diversidades dos indivíduos. Revista eletrônica Conhecimento em Destaque (Edição Especial), p. 1-10.

Zhu, J., Guo, M., Yang, T., Lai, X., Lei, Y. Y., He, M. L., \& Li, T. Y. (2017). Association between behavioral problems and gastrointestinal disorders among children with autism spectrum disorder. Chinese Journal of Pediatrics, 55(12), 905-910. 